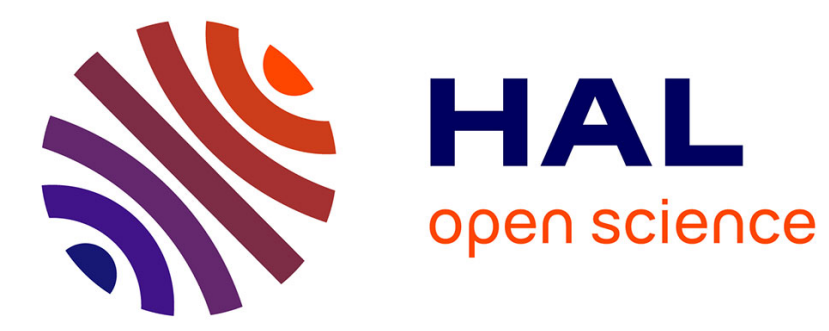

\title{
Fréedericksz transition on air
}

Juliette Plo, Dihya Sadi, Elio Thellier, Pawel Pieranski, Mehdi Zeghal, Patrick

Judeinstein

\section{- To cite this version:}

Juliette Plo, Dihya Sadi, Elio Thellier, Pawel Pieranski, Mehdi Zeghal, et al.. Fréedericksz transition on air. American Journal of Physics, 2021, 89 (6), pp.603-611. 10.1119/10.0003350 . hal-03234467

\section{HAL Id: hal-03234467 \\ https://hal.science/hal-03234467}

Submitted on 31 May 2021

HAL is a multi-disciplinary open access archive for the deposit and dissemination of scientific research documents, whether they are published or not. The documents may come from teaching and research institutions in France or abroad, or from public or private research centers.
L'archive ouverte pluridisciplinaire HAL, est destinée au dépôt et à la diffusion de documents scientifiques de niveau recherche, publiés ou non, émanant des établissements d'enseignement et de recherche français ou étrangers, des laboratoires publics ou privés. 


\title{
Fréedericksz transition on air
}

\author{
Juliette Plo, Dihya Sadi and Elio Thellier \\ ENS Paris-Saclay, 94235 Cachan, France
}

Pawel Pieranski* and Mehdi Zeghal

Laboratoire de Physique des Solides, Université Paris-Saclay, 91405 Orsay, France

Patrick Judeinstein

Laboratoire Léon Brillouin,CNRS-CEA, Université Paris-Saclay, 91191 Gif-sur-Yvette, France

(Dated: 10 décembre 2020)

The operational principle of twisted nematic displays involves the dielectric anisotropy of nematics. This crucial property was discovered about a hundred years ago by M. Jeżewski and W. Kast who used a so-called resonance method in which the frequency of an LC tank circuit was set by the capacitace of a capacitor filled with a nematic liquid crystal. Jeżewski and Kast observed that the resonance frequency changed upon application of a magnetic field to the capacitor. They interpreted the corresponding change in the dielectric permittivity as being due to reorientation of molecules by the magnetic field. Here, we describe a modern, simple and low-cost version of this experiment. Instead of the LC oscillator working with vacuum lamps, we use an op-amp RC oscillator in which a twisted nematic display plays the role of the capacitor. For the purpose of classroom demonstrations, the oscillator frequency $f_{R C}$ is detected by a software-defined radio operating in the double-side band mode (DSB). Upon an appropriate tuning of the reception frequency $f_{o}$, even small changes of $\Delta f=f_{R C}-f_{o}$ become audible. This setup is very convenient for demonstration and measurements of all characteristics of the Fréedericksz transition driven by magnetic or electric fields.

\section{INTRODUCTION}

\section{A. Discovery of the dielectric anisotropy of nematics by Jeżewski and Kast}

The dielectric anisotropy of nematics, crucial for functioning of twisted nematic displays, ${ }^{1,2}$ was discovered in 1924 by M. Jeżewski ${ }^{3}$ and W. Kast ${ }^{4}$. They used a resonance method, which consisted of using an LC tank circuit in which a plane capacitor $C_{s}$ filled with the paraAzoxyanisole (PAA) in the nematic state contributed to the total capacitance $C$, defining, with the inductance $L$, the resonance frequency $f=1 /(2 \pi \sqrt{L C})$. During measurements of the resonance frequency, Jeżewski and Kast discovered that upon application of a magnetic field orthogonal to the capacitor plates, the capacitance $C_{s}$ decreased. They interpreted the corresponding negative change of the dielectric permittivity $\epsilon$ as being due to a progressive orientation of "swarms" 5,6 of the PAA molecules caused by the growing magnetic field and concluded that the dielectric anisotropy $\epsilon_{a}=\epsilon_{/ /}-\epsilon_{\perp}$ of PAA was negative.

Today we know that the concept of swarms, thermally disordered in the absence of fields, is erroneous. This fact does not invalidate the determination of the dielectric anisotropy but unfortunately is also the reason that Jeżewski and Kast failed to discover a generic phenomenon, know today as the Fréedericksz transition, which was reported for the first time also in 1924 by V.K. Fréedericksz and A. Repieva. ${ }^{7}$

Today, the Fréedericksz transition is one of the most popular classroom experiments used for demonstrating the fascinating properties of nematic liquid crystals such

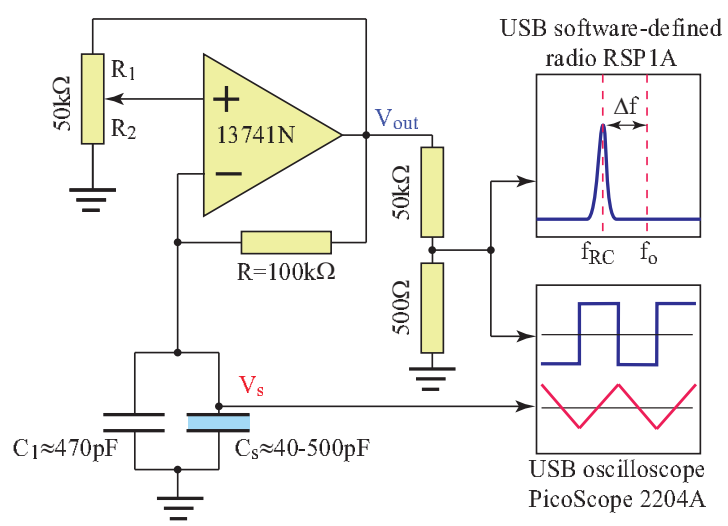

FiguRE 1. Modern classroom version of the Jeżewski-Kast setup. The frequency of the op-amp RC oscillator depends on the capacitance of the capacitor $C_{s}$ filled with nematic $5 \mathrm{CB}$. The oscillator signal is send to the software-defined radio which makes it audible and detects its frequency as a function of time.

as the optical, dielectric and magnetic anisotropies or the orientational elasticity and viscosity. For example, in the experiment described by Moses and Jensen, ${ }^{8}$ the Fréedericksz transition was induced by a magnetic field and detected optically from interferences of polarized light. When, during a lecture, the interference fringes are projected directly on a screen or imaged by means of a video camera, the Fréedericksz transition becomes visible as a motion of interference fringes.

In the present paper we propose a different "On Air" method which allows one not only to see but also to hear the Fréedericksz transition on a radio set. 

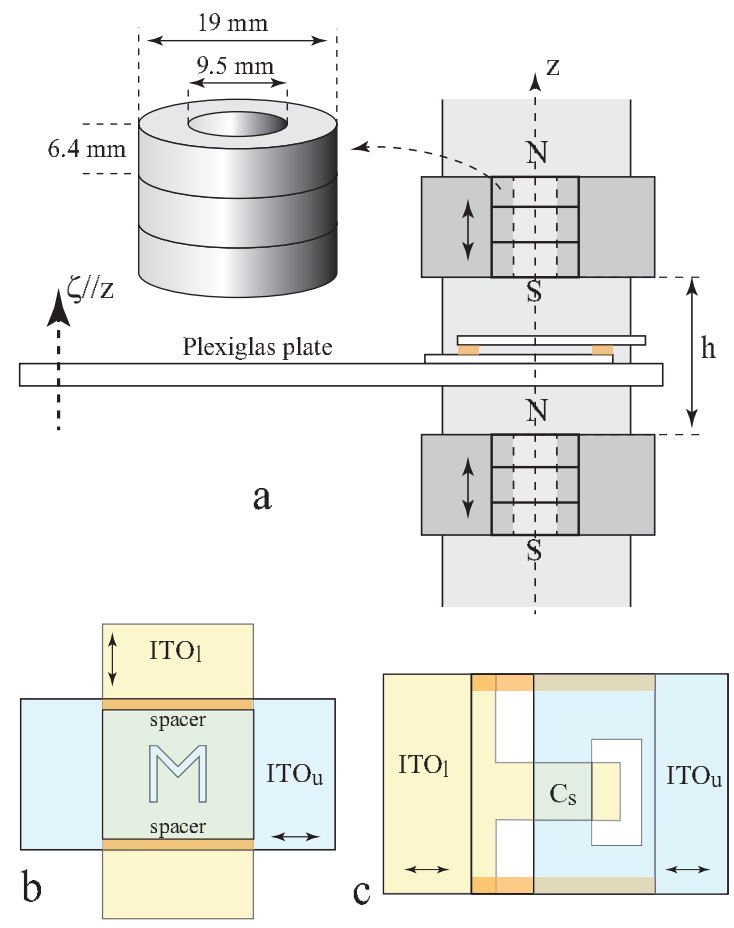

Figure 2. Experimental setup : a) Optical bench with two stacks of annular magnets mounted on translation stages. The nematic cell is supported by a plastic plate that can rotate around the axis $\zeta$. b) The twisted nematic cell with crossed planar anchorings. The letter M is etched in the ITO electrode on the upper glass slide. c) The nematic cell with parallel planar anchorings is used in experiments on the Fréedericksz transition in magnetic field. Its capacitance $C_{s}$ contributes to the period $T$ the RC oscillator (see Eq.1).

\section{B. Modern classroom version of the Jeżewski-Kast exepriment}

\section{Setup}

Inspired by the pioneering work of Jeżewski and Kast, we built a modern, classroom version of their experiment. Instead of the LC tank circuit excited by a vacuum lamp oscillator, we used an op-amp RC oscillator shown in Fig.1. Its frequency $f_{R C}$ depends on the capacitance $C_{s}$ of a flat capacitor (shown in Fig.2c) filled with 4-Cyano4'-pentylbiphenyl (5CB). The amplitude of the voltage $V_{s}$ across the sample is set with a potentiometer $R_{1}+R_{2}$ and is measured with an oscilloscope.

The oscillator frequency $f_{R C}$ is detected and displayed by means of a software-defined radio. ${ }^{10}$ When the radio operates in the double-sideband (DSB) mode, the frequency $f_{o}$ of the local oscillator can be tuned in such a manner that the frequency difference $\Delta f=f_{R C}-f_{o}$ becomes audible.

When the voltage $V_{s}$ across the nematic sample is larger than the threshold $V_{c}$ of the Fréedericksz transition ${ }^{7,9}$ (explained in the Appendix A), the director field of the
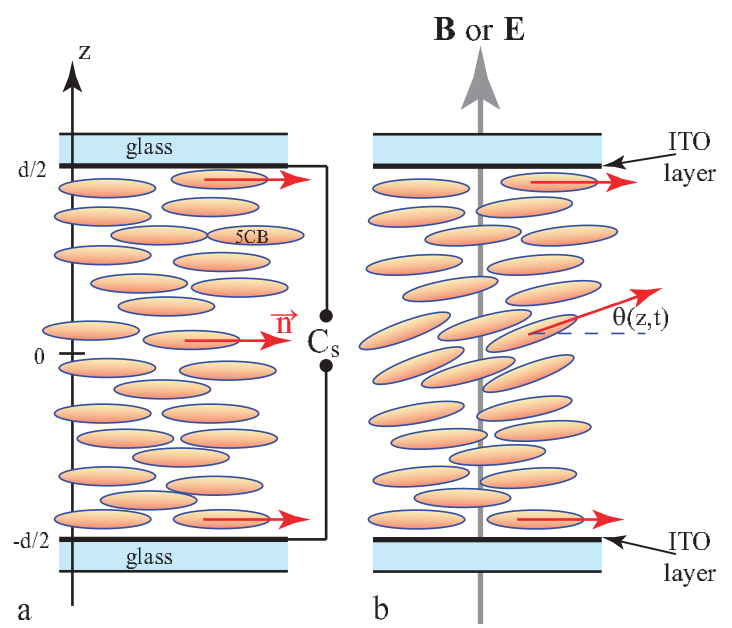

Figure 3. Principle of experiments. a) A cell, made of two glass slides coated with ITO electrodes and filled with a nematic $5 \mathrm{CB}$, plays the role of the capacitor $C_{s}$ in the $\mathrm{RC}$ opamp oscillator circuit (Fig.1). In the absence of fields, molecules of 5CB represented by ellipsoids (not to scale!) are on average parallel to each other, and their common orientation, represented by the unitary vector $\mathbf{n}$ called director, $\mathbf{n} / / \mathbf{x}$ is imposed by surfaces. b) In large enough electric or magnetic fields, the Fréedericksz transition occurs (see the Appendix A). The field-induced reorientation of molecules (i.e of the director) changes the mean dielectric permittivity $\langle\epsilon(\theta)\rangle \approx \epsilon_{\perp}+\epsilon_{a}\left\langle\theta^{2}\right\rangle / 2$. The corresponding change of the capacitance $C_{s}$ shifts the oscillation frequency $f$ detected by the SDR radio.

nematic is deformed as shown in Fig.3. The corresponding variation of the dielectric permittivity $\epsilon$ changes the capacitance $C_{s}$. The resulting variation of the frequency $f_{R C}$ is visible on the waterfall display of the SDR and is also audible as a change of the pitch. We can thus say that with our system the Fréedericksz transition becomes audible.

When $V_{s}$ is very small (of the order of $100 \mathrm{mV}$ ), the Fréedericksz transition can be induced by the magnetic field generated by two stacks of annular magnets ${ }^{11}$ shown in Fig.2a. By changing the distance $\mathrm{h}$ between magnets, supported by precision translation stages fixed on an optical bench, the intensity of the magnetic field can be controlled from 0 to $0.2 \mathrm{~T}$.

\section{Samples, nematic capacitors}

The samples, i.e. twisted nematic cells, were made of two glass slides $(25 \mathrm{~mm} \times 25 \mathrm{~mm} \times 1.1 \mathrm{~mm}$ or $75 \mathrm{~mm} \times 25 \mathrm{~mm} \times 1.1 \mathrm{~mm})$ coated with indium tin oxyde (ITO) electrodes. ${ }^{12}$ The distance between slides was set by mylar spacers of thickness $d=40 \mu \mathrm{m}$ or $110 \mu \mathrm{m}$ (see Figs.2b and c), and an epoxy glue, applied at lateral edges of the cell, kept the cell assembled (see Supplemental material). 
In quantitative measurements, it is necessary to locate the capacitor area in the center of the sample far from perturbing action of spacers and menisci. For this purpose, using scotch-tape masks and a $10 \%$ solution of $\mathrm{HCl}$ in water mixed with a small amount of a crude $\mathrm{Zn}$ powder, the ITO electrodes were etched into shapes of two orthogonal overlapping bands of width $8 \mathrm{~mm}$ shown in Fig.2c. By this means, the capacitor had a square shape of area $A=8 \mathrm{~mm} \times 8 \mathrm{~mm}$.

For visual demonstrations of the operational principle of twisted nematic displays (TND), a letter $\mathrm{M}$ was etched by the same method in the ITO electrode of one of the glass slides (see Fig.2b and Supplemental Material).

After the etching process, the slides were cleaned with acetone and wetted with a thin layer of a polyvinyl alcohol (PVA) solution in water. After drying at room temperature, the polymer layers on both slides were cured for $15 \mathrm{~min}$ at $120^{\circ} \mathrm{C}$ and buffed manually with a velvet fabric (other details are given in Supplemental Material). Surfaces treated by this method provided a planar anchoring of the nematic along the direction of the buffing.

Cells were assembled with parallel (Fig.2c) or crossed (Fig.2b) anchoring directions, using spacers and a small amount of an epoxy glue, and, finally, filled by capillarity with 5CB (other details are given in Supplemental Material). In principle, during construction of twisted nematic displays it is important to control the direction of the flow with the aim to avoid formation of domains with opposite chiralities. However, as the purpose of our experiments with the twisted nematic cell (see section IIIC) was also to explore the whole set of possible states of the TND, this requirement was unnecessary. The cell is supported by a horizontal Plexiglas plate which can rotate around the axis $\zeta$ parallel to the $\mathrm{z}$-axis and is located at a distance of $15 \mathrm{~cm}$ from it. By this means the sample can be very rapidly introduced in (or removed from) the magnetic field. The ITO electrodes are connected to the oscillator circuit by mechanical contacts with thin flexible brass blades.

\section{FRÉEDERICKSZ TRANSITION DRIVEN BY A MAGNETIC FIELD IN CELLS WITH PARALLEL ANCHORINGS}

\section{A. Frequency vs field, theoretical expectations}

In the limit of small amplitudes of the saw-tooth voltage $V_{s}\left(V_{s} \ll V_{\text {out }}\right)$, the period $T$ of the RC oscillator (see Fig.1) given by

$$
T \approx \frac{R_{2}}{R_{1}+R_{2}} 4 R\left(C_{1}+C_{s}\right)
$$

depends, via the capacitance $C_{s}$ of the nematic cell

$$
C_{s}=\epsilon_{o}\langle\epsilon(\theta)\rangle \frac{A}{d},
$$

on the deformation $\theta(z)$ of the director field by the magnetic field.

Close to the threshold $B_{c}$, the tilt angle of the director field can be approximated by

$$
\theta(z)=\theta_{o} \cos \left(\frac{\pi z}{d}\right)
$$

and, in the approximation of the isotropic elasticity, its amplitude $\theta_{o}$ is given by (see Eq.A4in Appendix A)

$$
\theta_{o}(B)^{2}=2\left(1-\frac{B_{c}^{2}}{B^{2}}\right)
$$

Knowing that

$$
\langle\epsilon(\theta)\rangle \approx \epsilon_{\perp}+\epsilon_{a} \frac{\left\langle\theta^{2}\right\rangle}{2}
$$

one obtains

$$
C_{s} \approx C_{s o}\left(1+2 \frac{\epsilon_{a}}{\epsilon_{\perp}} \frac{B-B_{c}}{B_{c}}\right)
$$

with

$$
C_{s o}=\epsilon_{o} \epsilon_{\perp} \frac{A}{d}
$$

Finally, using Eq.1 and Eq.6 one obtains

$$
f \approx f_{o}\left(1-2 \frac{C_{s o}}{C_{1}+C_{s o}} \frac{\epsilon_{a}}{\epsilon_{\perp}} \frac{B-B_{c}}{B_{c}}\right),
$$

with

$$
f_{o}=\frac{R_{1}+R_{2}}{R_{2}} \frac{1}{4 R\left(C_{1}+C_{s o}\right)} .
$$

In conclusion, in the limit of small distortions $\theta$ in the vicinity of the critical field $B_{c}$, the frequency of the oscillator should vary linearly with $B-B_{c}$.

\section{B. Frequency versus field, measurements}

$$
\text { 1. Small changes of } B, B>B_{c}
$$

Fig. 4 shows a typical display of the SDR window during experiments. Its upper half shows the instantaneous spectrum containing the peak corresponding to the first harmonic of the input signal. The lower half, called "waterfall", shows the variation of the peak frequency in time. It allows one to monitor the relaxation to equilibrium after changes of the magnetic field intensity. For example, the record on the waterfall window, shown in Fig.4, corresponds to the sequence $B=0.175 T \rightarrow$ $0.165 T \rightarrow 0.175 T$. Quantitative fitting showed that in this kind of experiment the two relaxation processes are exponential. 


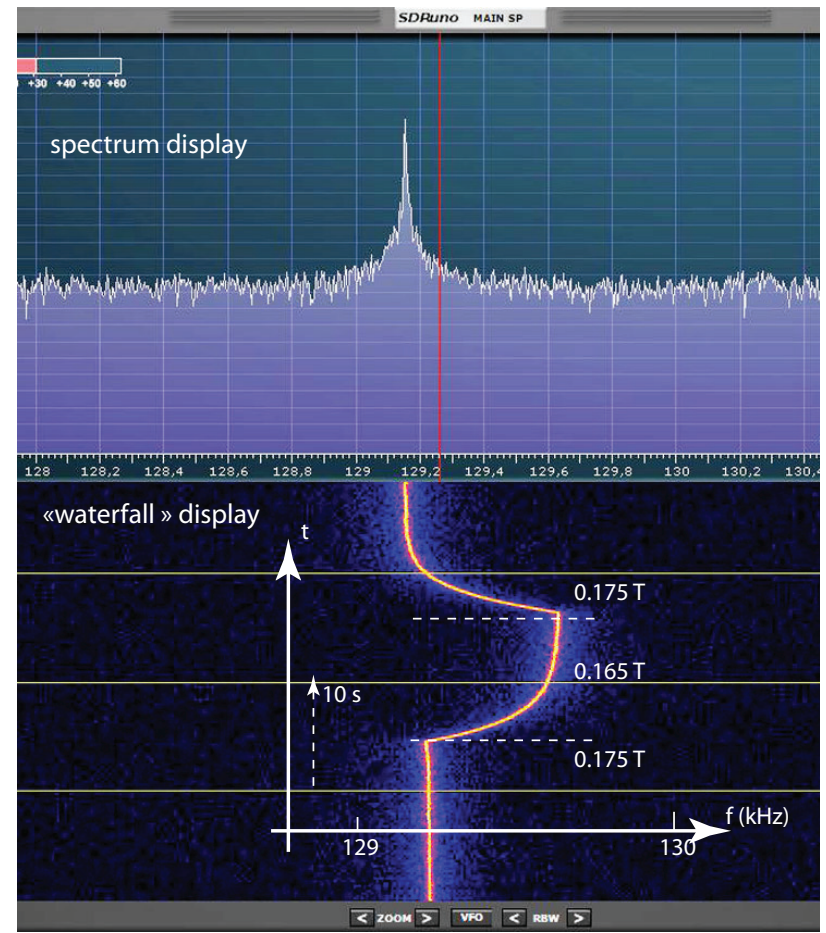

FiguRe 4. Typical variation of the RC oscillator frequency, recorded by means of the SDR, upon small changes of the field intensity B larger than the critical field $B_{c}=0.105 T$ of the $40 \mu \mathrm{m}$ nematic cell. The upper half of the window shows the instantaneous spectrum of the radio input signal showing the peak produced by the RC oscillator. The lower half, called "waterfall", shows temporal variation of the peak's frequency due to step-like changes of the magnetic field : $B=0.175 T \rightarrow$ $0.165 T \rightarrow 0.175 T$.

\section{Change from $B=0$ to $B>B_{c}$}

Relaxation toward the new equilibrium is more complex when the field $B>B_{c}$ is applied to the planar nematic cell free of distortion $(B=0)$. As explained above in section IB 2, this is done using the mobile plate supporting the sample. As an example, Fig.5 shows variation in time of $f$ recorded on the waterfall window after a sharp step rise of the magnetic field from $B=0$ to $B=0.114 T$. The most striking feature of this plot is its very slow initial rise. It is due to the fact that initially the director field is almost orthogonal to the magnetic field so that the magnetic torque proportional to $\theta$ is infinitesimal.

In the dipolar approximation of the magnetic field shown in Fig.11 of the Appendix B, the initial angle between the director (parallel to the $\mathrm{x}$ axis) and the magnetic field depends on the $z$ position of the sample represented by the dashed line $s$. In practice, the sample is always slightly out of the $z=0$ plane so that the magnetic field is slightly oblique with respect to the director field. As we will show later, this departure from the ideal orthogonal geometry not only determines the initial variation of $f$ with $t$ but also results in generation of a wall located at

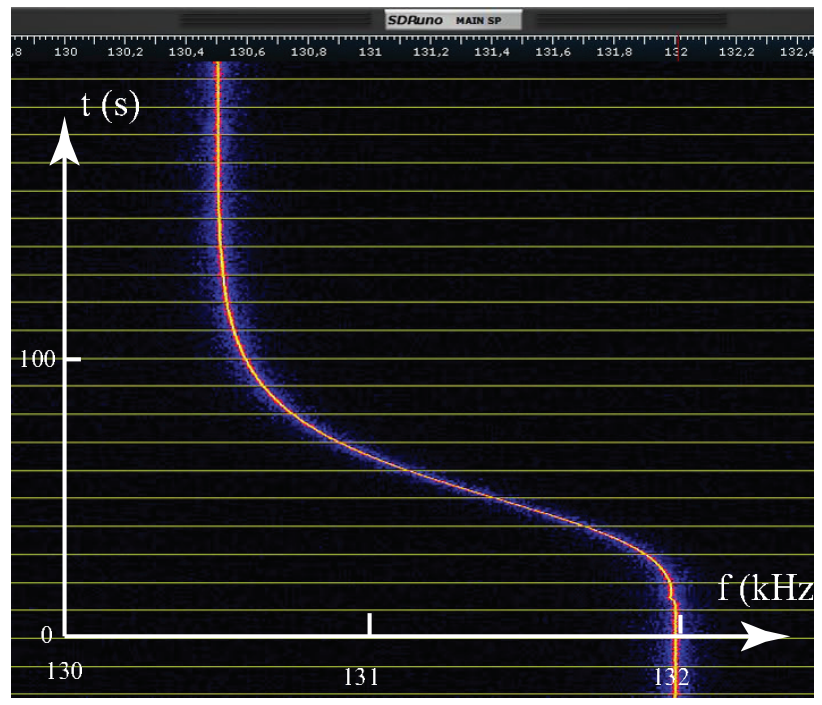

Figure 5. Typical variation of the RC oscillator frequency recorded by means of the waterfall display of the SDR radio after a step-like change the magnetic field, from zero to $\mathrm{B}=0.114 \mathrm{~T}>\mathrm{B}_{c}=0.105 \mathrm{~T}$, applied to the $40 \mu \mathrm{m}$ nematic cell.

$x=0$ and separating domains with $\theta>0$ and $\theta<0$.

\section{Determination of $B_{c}$}

Results of the two types of measurements, made with two samples of different thicknesses, are plotted in Fig.6. Fitting to the linear law given in Eq.8 was made using groups of experimental points limited by full circles. The critical field obtained from the fitting was $B_{c 40}=0.101 \mathrm{~T}$ for the cell assembled with $40 \mu \mathrm{m}$ spacers and $B_{c 110}=$ $0.052 \mathrm{~T}$ for the cell with $110 \mu \mathrm{m}$ spacers.

Theoretically (see Eq.A1-A6), the critical field given by

$$
B_{c}=\frac{\pi}{d} \sqrt{\frac{\mu_{o} K_{11}}{\chi_{a}}}
$$

should be inversely proportional to the sample thickness d. The ratio of critical fields measured with the two cells $B_{c 40} / B_{c 110}=1.94$ are slightly different from the expected one $: 110 / 40=2.75$. The most plausible explanation of this discrepancy is that real thicknesses of the two samples did not correspond exactly to the thicknesses of spacers. Presumably, the sample assembled with the $40 \mu \mathrm{m}$ spacers was thicker than expected.

\section{Measurements of the critical slowing down}

From a theoretical point of view, the Fréedericksz transition can be considered as a second-order phase transition during which the symmetry with respect to the 


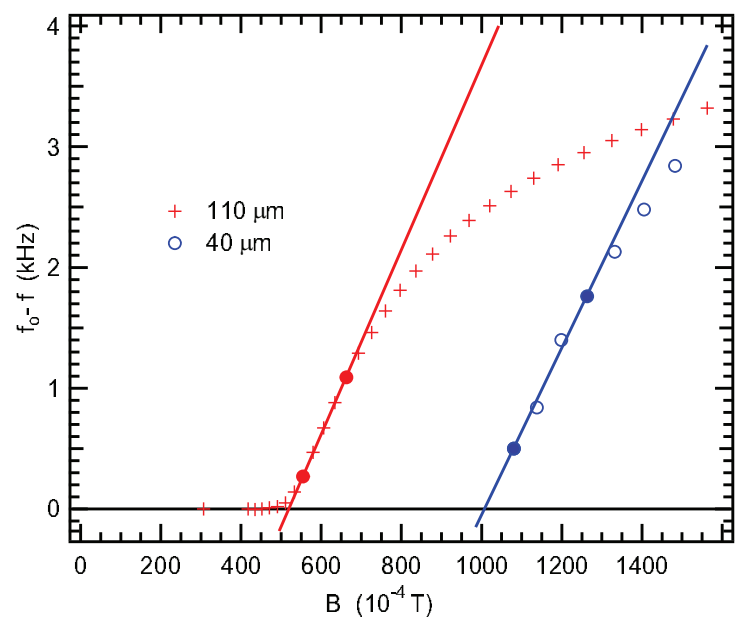

FiguRE 6. Fréedericksz transition in magnetic field. Determination of the critical field $\mathrm{B}_{c}$ from measurements, at equilibrium, of the resonance frequency $\mathrm{f}(\mathrm{B})$. As expected from Eq.8, the frequency shift $\delta f=f(0)-f(B)$ varies linearly with $B-B_{c}$ in the vicinity the threshold. The critical field $B_{c}=0.101 \mathrm{~T}$ for the cell assembled with $40 \mu \mathrm{m}$ spacers is about two times larger than $B_{c}=0.052 \mathrm{~T}$ for the cell with $110 \mu \mathrm{m}$ spacers.

$(x, z)$ mirror plane is broken. The order parameter resulting from this symmetry breaking is the tilt angle $\theta_{o}$. From From calculations in Appendix A we know that, in equilibrium, $\theta_{o}$ varies as $\sqrt{\left(B-B_{c}\right) / B_{c}}$. Moreover, on the basis of this analogy with second-order transitions, a critical slowing down of the relaxation time is expected in the vicinity of the critical field.

This theoretical prediction has been tested by a series of experiments in which transitions between two close equilibrium states, corresponding to fields $B$ and $B-\Delta B$, were recorded on the waterfall display of the SDR radio. Each experiment started by setting the intensity of the magnetic field to the value $B-\Delta B$, with $\Delta B \approx 0.003 \mathrm{~T}$. Once the equilibrium state was reached, the field was set rapidly to the nominal value $B$ (by vertical translation of one magnet) and the relaxation process was recorded with the waterfall display. In Fig.7a we show five such records. Relaxation times $\tau(B)$ were obtained by fits to an exponential law, and their inverses $1 / \tau(B)$ are plotted versus $B$ in Fig.7b.

As expected, $1 / \tau(B)$ is proportional to $B-B_{c}$, with $B_{c}=0.0536 \mathrm{~T}$. This "dynamical" value of the critical field is close to "static" one, $B_{c}=0.052 \mathrm{~T}$, obtained previously.
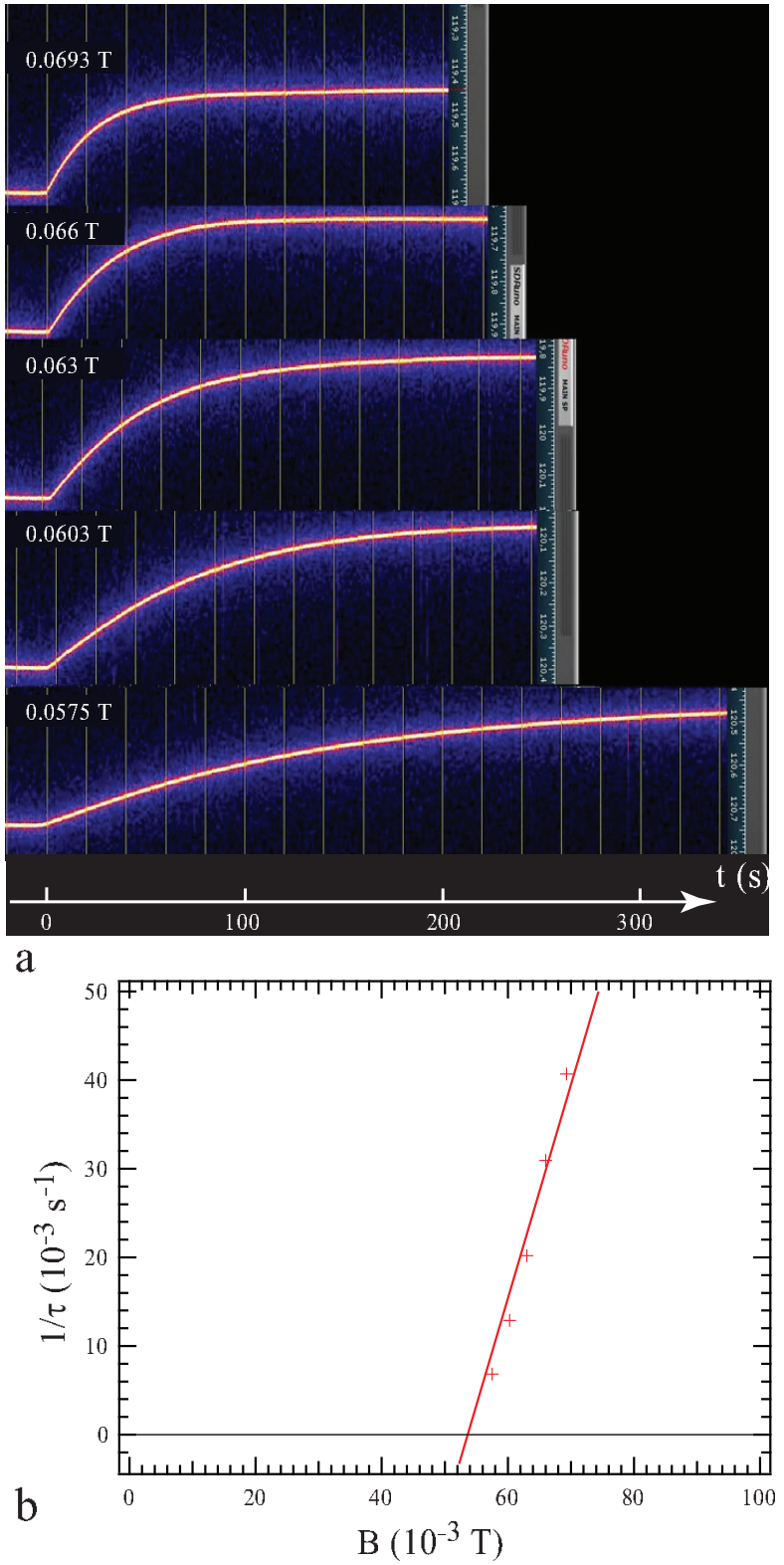

Figure 7. Critical slowing down in the vicinity of the Fréedericksz transition. a) The five plots $f(t)$ recorded with the waterfall display show that the relaxation time $\tau(\mathrm{B})$ increases when the threshold $\mathrm{B}_{c}$ of the Fréedericksz transition is approached from above. b) Plot of the inverse of the relaxation times determined from plots in (a). The fit to the linear law $1 / \tau \propto\left(B-B_{c}\right)$ gives $B_{c}=0.0536 \mathrm{~T}$.

\section{FRÉEDERICKSZ TRANSITION DRIVEN BY ELECTRIC FIELD IN TWISTED NEMATIC DISPLAY}

\section{A. Critical electric field, theory}

Our setup also allows for the study the Fréedericksz transition induced by an electric field. In this case the 
RC oscillator plays a dual role :

1. it generates the $\mathrm{AC}$ voltage $V_{s}$ driving the Fréedericksz transition

2. it serves as a probe, detecting the Fréedericksz transition through changes of the $\mathrm{AC}$ frequency.

In other words, in our setup, self-detection of the Fréedericksz transition occurs. The Fréedericksz transition takes place when the applied electric field $E=V_{s} / d$ is larger than the critical one $E_{c}$. The critical electric field $E_{c}$ for a planar nematic sample can be calculated in exactly the same manner as $B_{c}$ in the Appendix A by replacing the magnetic torque term in Eq.A1 by the electric one

$$
\Gamma_{\text {elec }}=\epsilon_{o} \epsilon_{a} E^{2} \sin \theta \cos \theta .
$$

With this substitution, one obtains

$$
E_{c}=\frac{\pi}{d} \sqrt{\frac{K_{11}}{\epsilon_{o} \epsilon_{a}}} .
$$

Because $E_{c} \propto 1 / d$, the critical voltage $V_{c}=E_{c} d$ for the Fréedericksz transition in a planar nematic cell does not depend on the sample thickness.

In the case of the twisted nematic display shown in Fig.2b, the calculation of the critical field is more complex (see e.g. ref. ${ }^{13}$ or the textbook on liquid crystals by $\mathrm{S}$. Chandrasekhar ${ }^{14}$ ) and leads to the expression

$$
V_{c}=\pi \sqrt{\frac{K_{11}+\left(K_{33}-2 K_{22}\right) / 4}{\epsilon_{o} \epsilon_{a}}}
$$

involving three Oseen-Frank elastic constants. In practice, the ON state of the Twisted Nematic Display requires application of a voltage at least two times larger than $V_{c}$.

\section{B. Self-detection of the critical voltage of the TND}

The critical voltage $V_{c}$ of the TND was measured with our setup in the following manner. First, the (RMS) voltage $V_{s}$ on the capacitor $C_{s}$ of the RC oscillator was progressively increased by steps, by means of the potentiometer $R_{1}+R_{2}$. As long as $V_{s}<V_{c}$, the oscillator frequency remains constant because the capacitance of the sample does not change $\left(C_{s}=C_{s o}\right)$. On the contrary, when $V_{s}$ is larger then the threshold value $V_{c}$, the oscillator frequency changes because the capacitance $C_{s}$ of the nematic cell decreases when the tilt angle $\theta_{o}(t)$ increases.

Once the approximate value of $V_{c}$ is known, the voltage $V_{s}$ on the capacitor $C_{s}$ of the RC oscillator is set to some value larger than $V_{c}$. The RC oscillator is then shut down (by disconnecting the $+9 \mathrm{~V}$ and $-9 \mathrm{~V}$ supplies of the op-amp) and the sample is allowed to relax for several minutes.

After this relaxation stage, the $\mathrm{RC}$ oscillator is switched on at $t=t_{o}$ and the spectrum of its output signal is recorded as a function of time with the waterfall display of the SDR. A typical record obtained with $V_{s}=1200 \mathrm{mV}$ (RMS) is shown in Fig.8. For for such a large amplitude $V_{s}$, the frequency of the fundamental mode $f_{1}=7.5 \mathrm{kHz}$ is much smaller than $f_{1}=132 \mathrm{kHz}$ in Fig.5 where $V_{s}$ was about $100 \mathrm{mV}$ (RMS).

As the output signal of the RC oscillator is a square wave, its spectrum contains many harmonics. The waterfall display below the spectrum shows that the Fréedericksz transition occurred : variation in time of all frequencies $f_{n}(t)$ is similar to the one of the fundamental mode in Fig.5 discussed previously. For the fifth mode, its initial frequency at $t=0$ is $f_{5}(0)=38.5 \mathrm{kHz}$. After the evolution displayed on the waterfall window, the frequency of the fifth mode converges to its new equilibrium value $f_{5}(\infty)=31 \mathrm{kHz}$. The relative change of the frequency can be expressed as a dimensionless parameter $\delta=1-f_{5}(\infty) / f_{5}(0)$.

The same experiment was repeated with several amplitudes $V_{s}$ and the results are summarised in Fig.9. The value of the critical voltage $V_{c}=0.750 \mathrm{mV}$ (RMS) is obtained from a linear fit $\delta \propto\left(V_{s}-V_{c}\right)$.

\section{Symmetries of four ON states in a twisted nematic display}

The operational principle of the twisted nematic display involves its action on the polarization of the transmitted light.

In the OFF state, linear polarization of the input beam follows the helical configuration of the director that rotates by 90 degrees around the $z$ axis between the two glass slides. Therefore the output polarisation is orthogonal to the input one. When the TND is located between crossed polarizers the input light is transmitted. We can thus say that with crossed polarizers the OFF state (i.e. with $V_{s}$ ) is bright.

In the ON state, the input linear polarization remains almost unchanged so that input light is not transmitted. The ON state is dark.

In the case of the TND shown in Fig.2b, the letter M was etched in one of the ITO electrodes. Therefore, the electric field is not applied in this M-shaped area so that we expect that it remains in the OFF bright state.

This is the case in the picture of Fig.10a where the letter $\mathrm{M}$ is bright. This picture obtained with slightly uncrossed polarizers reveals however that there are not one but four different ON states of different shades (please, consult the online version) :

1. dark green

2. bright green

3. dark violet

4. bright violet

Fig.10b explains that the green and violet areas correspond to respectively the right and left helicities of the director field $\mathbf{n}(\mathrm{z})$. These areas of opposite helicities in 


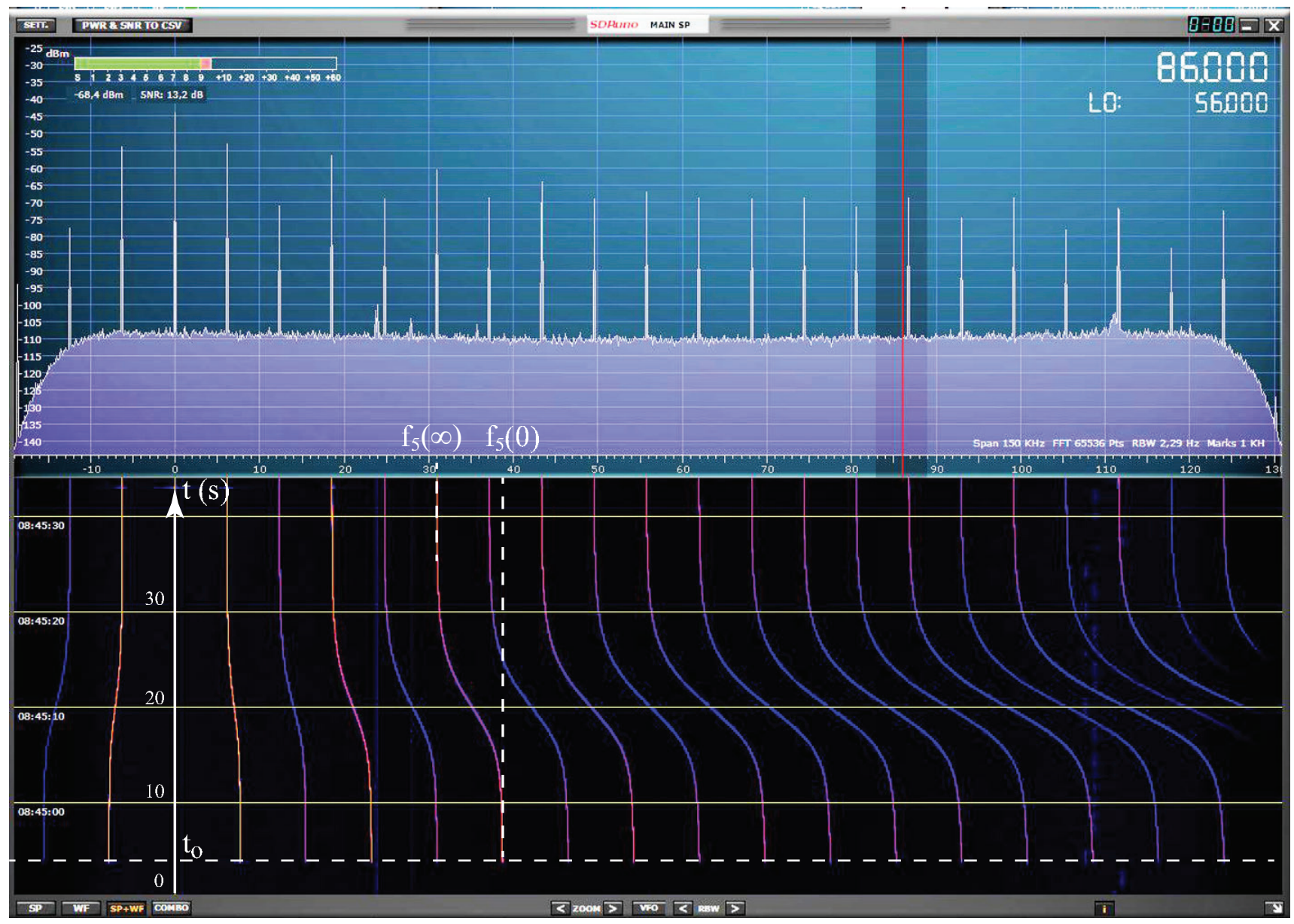

Figure 8. Evolution of the spectrum of the output signal $V_{\text {out }}$ detected by the SDR radio. At $t=t_{o}$ the RC oscillator has been switched on and the voltage $V_{s}=1200 \mathrm{mV}$ (RMS) has been applied to the sample. For such a large amplitude, the frequency $f_{1}=7.5 \mathrm{kHz}$ of the fundamental mode is much smaller then $f_{1}=132 \mathrm{kHz}$ in Fig.5. As the output signal of the RC oscillator is a square wave, its spectrum contains many harmonics. The waterfall display below shows that the Fréedericksz transition occurs : variation in time of all frequencies $f_{n}(t)$ is similar to the one of the fundamental mode in Fig.5.

Fig.10a are separated by black lines labeled "disclination". The central scheme in Fig.10b (see also Fig.16 in Supplemental Material) shows that, in three dimensions, these lines are located halfway between the glass slides of the TND $(z=0)$ and have a remarkable property : on a circuit (drawn with a dotted line) surrounding one of them, the director $\mathbf{n}$ rotates by $\pi$. Moreover, this rotation by $\pi$ is conserved when the size of the circuit shrinks to zero. Therefore, orientation of the director $\mathbf{n}$ must be undetermined on the line itself. Therefore, in mathematical sense, disclinations are linear topological singularities of the director field $\mathbf{n}(x, y, z)$.

Before filling with liquid crystal, the symmetry of the empty twisted cell is quite high. It contains mirror planes $(x, z)$ and $(y, z)$ as well as two-fold axes $\xi$ and $\zeta$. After filling, the mirror symmetries are broken by the right and left helicities of the director field.

A second symmetry breaking occurs at the Fréedericksz transition : the up or down tilts of the director $(\theta>0$ or $\theta<0)$ break the two-fold symmetries. In Fig.10a bright and dark shades of the green and violet colors are due to the up and down tilts of the director field. They are separated by white lines called "walls".

\section{CONCLUSION}

Liquid crystal physics lies at the intersection of many fields ranging from electromagnetism to statistical physics through optics. It is therefore not surprising that these fascinating systems are increasingly taught in undergraduate courses on soft matter physics. ${ }^{15-17}$

During lectures on liquid crystals, many classroom experiments can be performed ${ }^{18-21}$. Among them are demonstrations of the Fréedericksz transition, leading to the operational principle of twisted nematic displays. Traditionally, distortions of the director field were indu- 


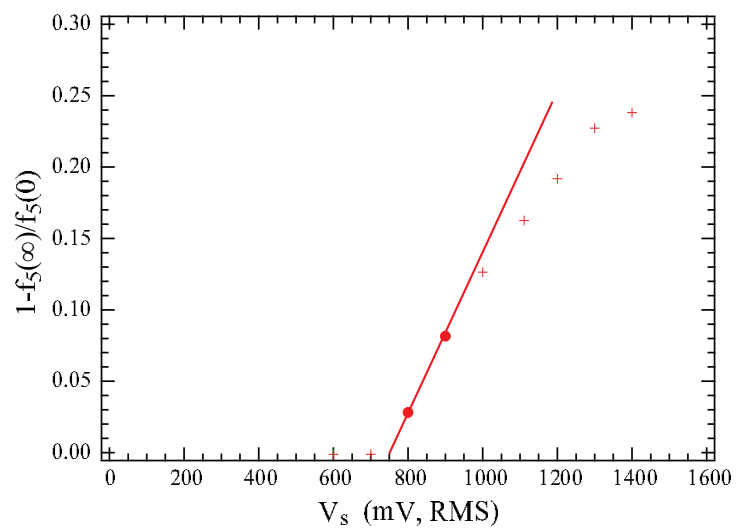

FiguRE 9. Fréedericksz transition driven by the electric field in the twisted nematic display. The dimensionless parameter $\delta=1-f_{5}(\infty) / f_{5}(0)$ represents the relative variation of the frequency : $f_{5}(0)$ and $f_{5}(\infty)$ are frequencies of the fifth harmonic measured respectively at $t=0$ without distortion and in the new equilibrium reached after a sufficiently long time.

ced by the magnetic field of an electromagnet and were detected optically from interference fringes in polarized He-Ne laser light. The experiment reported here benefited from recent availability of low-cost neodymium magnets and software defined radios (see the Supplemental Material). Construction of the very simple op-amp RC oscillator powered by $9 \mathrm{~V}$ batteries is easy. Construction of nematic cells, twisted or not, is less straightforward but remains possible thanks to detailed explanations given in the Supplemental Material. Twisted nematic displays can also be obtained by deconstruction of digital clocks and can be used for demonstrating the Fréedericksz transition in the electric field. However, due to their very small thickness (a few $\mu \mathrm{m}$ ) their response, to the application or variation of the electric field, is too rapid for measures with the SDR radio.

Using a SDR as a detector of the signal produced by the $\mathrm{RC}$ oscillator has at least three advantages :

1. frequency variations $\Delta f(B)$ of the order of a few $\mathrm{kHz}$ due to the Fréedericksz transition can be detected with precision of the order of $10 \mathrm{~Hz}$,

2. variations of the frequency $f$ in time can be easily recorded and analysed; this allows one to study the dynamics of the Fréedericksz transition,

3. as already emphasized in the Introduction, in the double-sideband mode of the SDR, the Fréedericksz transition is audible as a change of the pitch; this is desirable during lectures for a large audience in an amphitheater.

Finally, we note that experiments performed with the setup described here raised a new interesting issue : can one control generation of the four possible ON states of a twisted nematic display? Knowing that these four states are separated by disclinations and walls, this can be rephrased as : can one control generation of disclinations

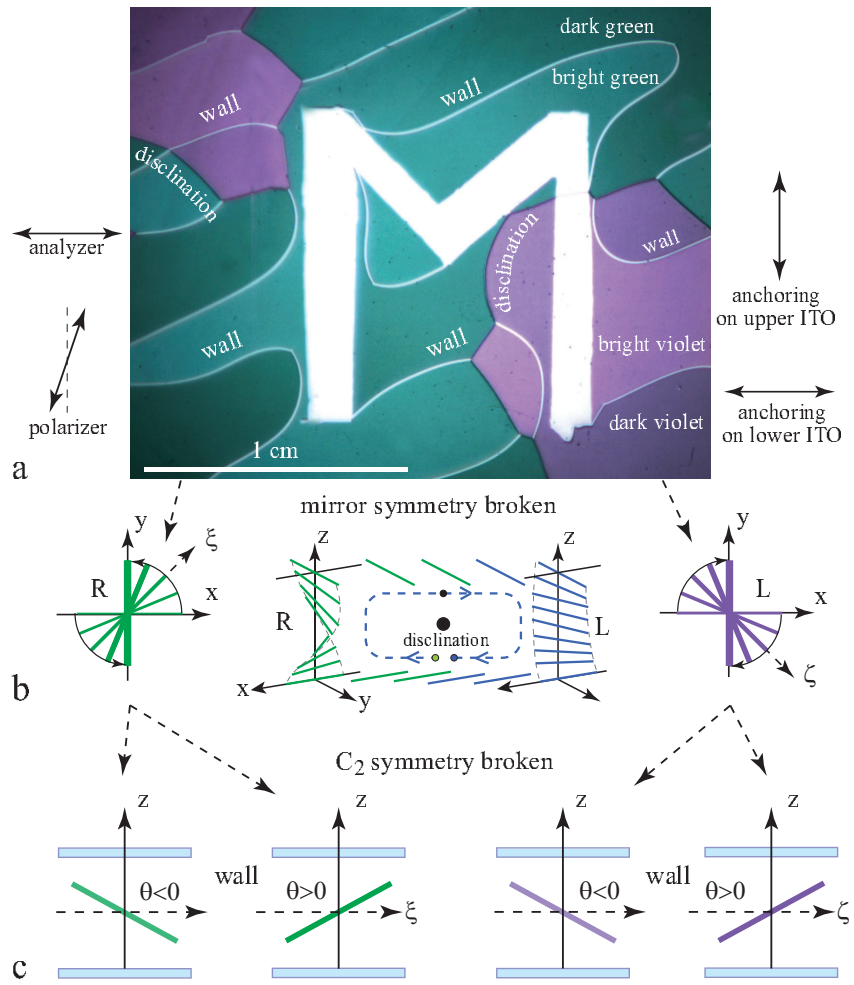

Figure 10. Four possible states "ON" of a twisted nematic display (TND) resulting from two successive symmetry breakings. a) View of the TND between slightly uncrossed polarizers. The green and violet areas correspond respectively to the two possible textures of opposite chirality resulting from the breaking of the mirror symmetry represented in b. b) The right-hand $(\mathrm{R})$ and left-hand $(\mathrm{L})$ twisted textures in TND differ by orientations, $\xi$ or $\zeta$, of the director $\mathbf{n}$ halfway $(z=0)$ between slides with $\mathrm{x}$ and $\mathrm{y}$ anchoring directions. The perspective view in the centre shows that domains with opposite chiralities are separated by singular lines. c) Second symmetry breaking. The two-fold symmetry of the L and R chiral textures, with respect to the $\xi$ and $\zeta$ axes, is broken at the Fréedericksz transition. In the picture a, domains with $\theta>0$ and $\theta<0$ have different green and violet shades and are separated by white walls $\left(V_{s}=2.1 \mathrm{~V}\right.$ RMS $)$.

and walls in twisted nematic cells? The answer to this question is "yes" but it is beyond the scope of the present paper. We intend to publish it soon.

\section{Annexe A: Theory of the Fréedericksz transition}

The equation of motion for the tilt angle of the director in the cell without twist contains three terms corresponding to the elastic, magnetic and viscous torques (per unit volume $)^{9,21}$

$$
K_{11} \frac{\partial^{2} \theta}{\partial z^{2}}+\frac{\chi_{a}}{\mu_{o}} B^{2} \sin \theta \cos \theta-\gamma_{1} \frac{\partial \theta}{\partial t}=0 .
$$



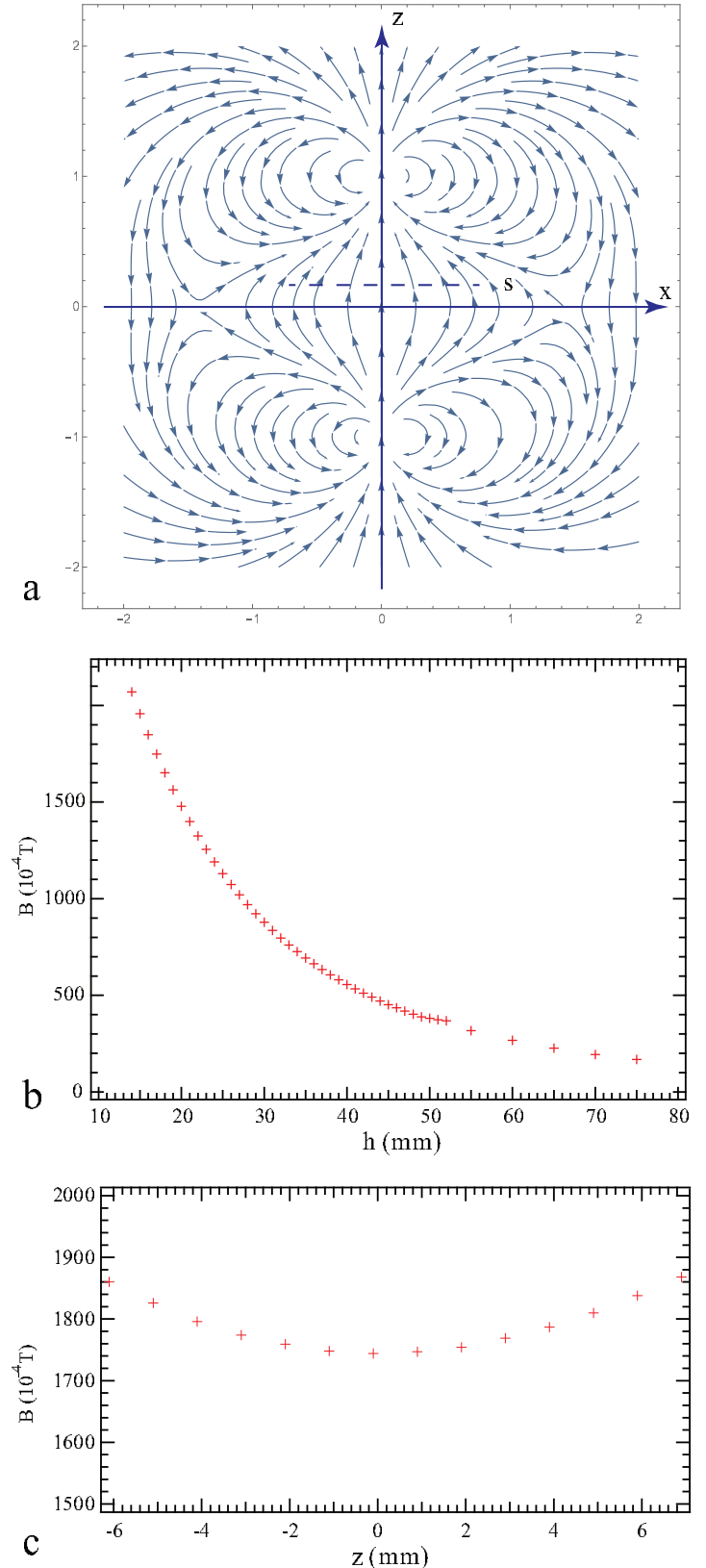

Figure 11. Characteristics of the magnetic field produced by the two stacks of annular neodymium magnets of the setup shown in Fig.2. a) Magnetic field calculated in dipolar approximation by a Mathematica program given in Supplemental Material. The dashed line s represents the position of the sample slightly above the symmetry plane $(x, y)$. b) Field dependence on the distance $\mathrm{h}$ between supports of the cylindrical magnets. Measures made with a Hall-probe magnetometer (Walker Scientific MG-5D). c) Field dependence on the distance $z$ from the symmetry plane $(x, y)$, measured with a Hall-probe magnetometer.
In the limit of small deformations, i.e. close to the threshold $B_{c}$, after expansion of the second term in a power series of $\theta$ one obtains

$$
K_{11} \frac{\partial^{2} \theta}{\partial z^{2}}+\frac{\chi_{a}}{\mu_{o}} B^{2}\left(\theta-\frac{2}{3} \theta^{3}\right)-\gamma_{1} \frac{\partial \theta}{\partial t}=0 .
$$

When $\theta(z, t)$ is expanded in a Fourier series, its first term, satisfying the boundary conditions $\theta(z= \pm d / 2)=0$, is

$$
\theta(z, t)=\theta_{o}(t) \cos \left(\frac{\pi z}{d}\right) .
$$

In equilibrium, i.e. when $\partial \theta / \partial t=0$, substitution of $\mathrm{A} 3$ in $\mathrm{A} 2$, followed by expansion of the term $\cos ^{3}(\pi z / d)$ into Fourier series limited to the first term (A3), leads to

$$
\theta_{o}\left(1-\frac{\theta_{o}^{2}}{2}-\frac{B_{c}^{2}}{B^{2}}\right)=0
$$

with

$$
B_{c}=\frac{\pi}{d} \sqrt{\frac{\mu_{o} K_{11}}{\chi_{a}}}
$$

For $B<B_{c}$, the first solution of the Eq.A $4, \theta_{o}=0$ is stable while for $B>B_{c}$ the second one has to be taken and one obtains

$$
\theta_{o}(B)= \pm 2 \sqrt{\frac{B-B_{c}}{B_{c}}}
$$

\section{Annexe B: Field generated by two stacks of annular magnets}

The field generated by the two stacks of annular magnets (see Fig.2a) is quite complex. For the purpose of the present paper it suffices to know its approximate geometry, represented in Fig.11a, which was calculated numerically in the dipolar approximation. For symmetry reasons, $\mathbf{B}$ is orthogonal to the sample in the $z=0$ plane. The field intensity B measured in this symmetry plane as a function of the distance $\mathrm{h}$ between supports of the magnets is plotted in Fig.11b. Finally, in Fig.11c we show how the field intensity $B(0,0, z)$ varies with the distance $\mathrm{z}$ from this symmetry plane.

\section{ACKNOWLEDGMENTS}

PP is grateful to organizers of the 15th European Conference on Liquid Crystals in Wroclaw for the invitation. During preparation of a talk on "Liquid Crystals in Poland" the crucial contributions of M. Jeżewski and W. Kast came to light. The idea of using a software defined radio as a detector was suggested to PP by Piotr Pieranski. This work benefited from the technical assistance of I. Settouraman, V. Klein, S. Saranga, J. Sanchez, M. Bottineau, J. Vieira, I. Nimaga and C. Goldmann. PP thanks S. Ravy for a financial support. We thank also A. Thiaville and S. Rohart for the loan of the magnetometer. 
* pawel.pieranski@u-psud.fr

1 P.J. Collings, "Liquid crystal displays", Am. J. Phys. 63, 1044-1048 (1995).

2 P.J. Collings and J.W Goodby, Introduction to liquid crystals : chemistry and physics (Crc Press 2019).

${ }^{3}$ M. Jeżewski, "Influence du champ magnétique sur les constantes diélectriques des cristaux liquides," J. de Physique et Radium 5 (2), 59-64 (1924).

${ }^{4}$ W. Kast, "Anisotropie der flüssigen Kristalle bezüglich ihrer Dielektrizitätkonstanten und ihrer elektrischen Leitfähigkeit", Annalen der Physik, 378 (1-2), 145-160 (1924).

5 Swarms' model of liquid crystals : In the absence of fields, elongated molecules of liquid crystals were supposed to be parallel each to other only locally in small domains, about $1 \mu \mathrm{m}$ in size, called "swarms". The thermally disordered ensemble of swarms was supposed by Jeżewski and Kast to be oriented progressively by a growing magnetic field.

${ }^{6}$ L.S. Ornstein and W. Kast, "New arguments for the swarm theory of liquid crystals", Transactions of the Faraday Society, 29, 930-944 (1933).

${ }^{7}$ V. K. Fréedericksz und A. Repieva ,"Theoretisches und Experimentelles zur Frage nach der Natur der anisotropen Flüssigkeiten", Zeitschrift für Physik, 42(7), 532-546 (1927).

8 T. Moses and B. Jensen "The Fréedericksz transition in liquid crystals : An undergraduate experiment for the advanced laboratory", Am. J. of Phys. 66, 49-56 (1998).

9 a) P.-G. de Gennes, The Physics of Liquid Crystals, first edition (Oxford University Press 1974)

b) P.-G. de Gennes and J. Prost, The Physics of Liquid Crystals, second edition (Oxford University Press 1993).
10 The idea of using a software defined radio (SDR) for classroom experiments stems from Piotr Pieranski. In experiments reported here we used the model RSP1A from SDRplay. RSP1A combined with the windows-based SDRuno software (freeware available from SDRplay) covers the RF spectrum from $1 \mathrm{kHz}$ to $2 \mathrm{MHz}$.

11 The neodymium annular magnets were purchased from Supermagnete.

12 The ITO coated glass slides, $25 \mathrm{~mm} \times 25 \mathrm{~mm} \times 1.1 \mathrm{~mm}$ and $75 \mathrm{~mm} \times 25 \mathrm{~mm} \times 1.1 \mathrm{~mm}$, were purchased from SigmaAldrich. Thin indium tin oxide coatings are conducting and transparent.

13 K.H. Yang, "Fréedericksz transition of twisted nematic cells" App. Phys. Lett. 43, 171-173 (1983).

14 S. Chandrasekhar, Liquid Crystals, page 106-113, 2nd edition (Cambridge University Press, 1992).

15 R.A.L. Jones, Soft Condensed Matter, (Oxford Univrsity Press, Oxford 2002).

${ }^{16}$ M. Kleman and O.D. Lavrentovich, Soft Matter Physics, (Springer-Verlag, New York 2003).

17 I.W. Hamley, Introduction to Soft Matter, (Wiley, New York 2007).

18 J. Pavlin, N. Vaupotič and M. Čepič, "Direction dependence of the extraordinary refraction index in uniaxial nematic liquid crystals", European Journal of Physics 34, 331-344 (2013).

19 J. Pavlin, N. Vaupotič and M. Čepič, "Liquid crystals : a new topic in physics for undergraduates", European Journal of Physics, 34, 745-761 (2013).

20 H.-S. Kitzerow and C. Bahr, Chirality in Liquid Crystals, chapter Classroom experiments with liquid crystals, (Springer 2001).

21 P. Oswald and P. Pieranski, Nematic and Cholesteric Liquid Crystals, Concepts and physical properties illustrated by experiments, (Taylor \& Francis, 2005). 